\title{
Rhabdomyoma association with atrioventricular septal defect in an infant: a rare coincidence
}

\author{
Ilker Mercan ${ }^{1}$ (i) $\cdot$ Muhammet Akyüz ${ }^{1}$ (i) $\cdot$ Onur Isık $^{1}$ (i)
}

Received: 17 May 2020 /Revised: 21 June 2020 / Accepted: 6 July 2020 / Published online: 21 July 2020

(C) Indian Association of Cardiovascular-Thoracic Surgeons 2020

\begin{abstract}
Cardiac rhabdomyoma is the most common primary heart tumor in childhood. This tumor, which is frequently associated with tuberous sclerosis complex, mostly disappears in childhood with spontaneous regression. Surgical resection is required in case of outflow obstruction and arrhythmia and when protruding to disrupt the filling of the heart cavities. There are very few case series in the literature about rhabdomyoma, whose relationship with other congenital heart defects has not been clearly verified. In this study, we report our approach to the tumor during the corrective surgery of the infant, who was diagnosed with an atrioventricular septal defect and patent ductus arteriosus, and rhabdomyoma accompanying these malformations. We treated this asymptomatic rhabdomyoma with everolimus in line with the current literature, without excision.
\end{abstract}

Keywords Rhabdomyoma $\cdot$ Infant $\cdot$ Atrioventricular septal defect

\section{Introduction}

Cardiac rhabdomyoma is the most common primary heart tumor detected in infancy and childhood. The clinical course is generally benign. In reported studies, spontaneous regression was observed in the majority of cases. The relationship of this tumor, which was first described in 1862, with tuberous sclerosis (TS) is well established [1]. Although it has a good clinical course, surgical resection is inevitable when it causes ventricular outflow/inflow obstruction, myocardial dysfunction, or malignant arrhythmias.

Despite many studies, very few congenital heart diseases have been reported to be associated with cardiac rhabdomyoma. There are studies that argue that rhabdomyoma may lead to any defect by causing a disruption in the embryological development steps of the heart. However, it is still controversial whether these two cardiac pathologies emerge as different pathologies [2]. In this case study, we report a rare case of TS with cerebral,

Ilker Mercan

drilkermercan@hotmail.com

1 Department of Pediatric Heart Surgery, Izmir University Of Health Sciences Tepecik Training And Research Hospital, Sanayi Caddesi No:7 Bornova, İzmir, Turkey dermatological, and renal lesions associated with cardiac rhabdomyoma and atrioventricular septal defect (AVSD).

\section{Case report}

A female child was referred to our clinic with symptoms of congestive heart failure, mild pulmonary hypertension (pulmonary artery pressure, $30 \mathrm{mmHg}$ ), and resistant to medical treatment. The patient was 3 months old and with $3.800 \mathrm{~g}$ of weight. During the examination, S1 hard, S2 double, grade 3/ 6 pansystolic murmur was heard in the lower left sternal region. The electrocardiogram was a sinus rhythm at 150 beats/ min with left axis deviation. The patient had growth retardation and tachypnea with an oxygen saturation of $95 \%$ on room air. On chest $\mathrm{x}$-ray, the cardiothoracic ratio was 0.6 and pulmonary plethora was seen. The patient had no signs of Down syndrome.

On 2D transthoracic echocardiographic (TTE) examination, 7-mm primum atrial septal defect (ASD) in the interatrial septum and 5-mm ventricular septal defect (VSD) in the interventricular septum were observed. There was a second degree of insufficiency in the left atrioventricular (AV) valve commensurate with atrioventricular septal defect (AVSD) transitional type. At the base of the interventricular septum, in close proximity to the membranous septum, a uniformly limited hyperechogenic mass of $1.5 \times 1.0 \mathrm{~cm}$ was observed 
in the membranous septum. This mass minimally protruded into the cavity of the right ventricle (Fig.1). Doppler examination did not reveal any evidence of inflow or ventricular outflow tract obstruction. Patent ductus arteriosus (PDA) was observed and $6 \mathrm{~mm}$ in diameter. Cardiac mass seen by echocardiography was evaluated in more detail using multidetector computed tomography (MDCT) (Fig.2).

With the diagnosis of rhabdomyoma, the patient was examined for advanced TS. With cerebral imaging, cortical tubers and subependymal nodules were observed. Multiple renal cysts were detected using urinary ultrasonography. In the dermatological examination, hypomelanotic macules and angiofibromas were detected, and the diagnosis of TS was confirmed. These TS findings and the typical TTE and MDCT appearance of the mass (echogenic, nodular, ventricular embedded in the myocardium, protruding to the right ventricle, limited, echogenic, nodular) clarified the diagnosis of rhabdomyoma [3]. The patient's condition was discussed with pediatric cardiologists and surgical intervention was planned.

Arterial cannulation from the ascending aorta and venous cannulations from both vena cava were performed. Double ligation and division of the ductus arteriosus was performed. Cardioplegia was administered antegradely from the aortic root. Right atriotomy was carried out. Both primum ASD $(7 \mathrm{~mm})$ and secundum ASD $(3 \mathrm{~mm})$ were observed in the interatrial septum (IAS). The left atrial vent was put through secundum ASD. Atrioventricular septal defect repair was done using a modified single patch technique. On the right ventricular side of the interventricular septum (IVS), on the membranous septum, adjacent to the tricuspid valve anteroseptal commissure, there was a well-circumscribed mass with shiny surface of the primary tumor (Fig.3). In addition, bright, scattered lesions of a millimeter size were observed on the surface of the tricuspid valve. However, it was

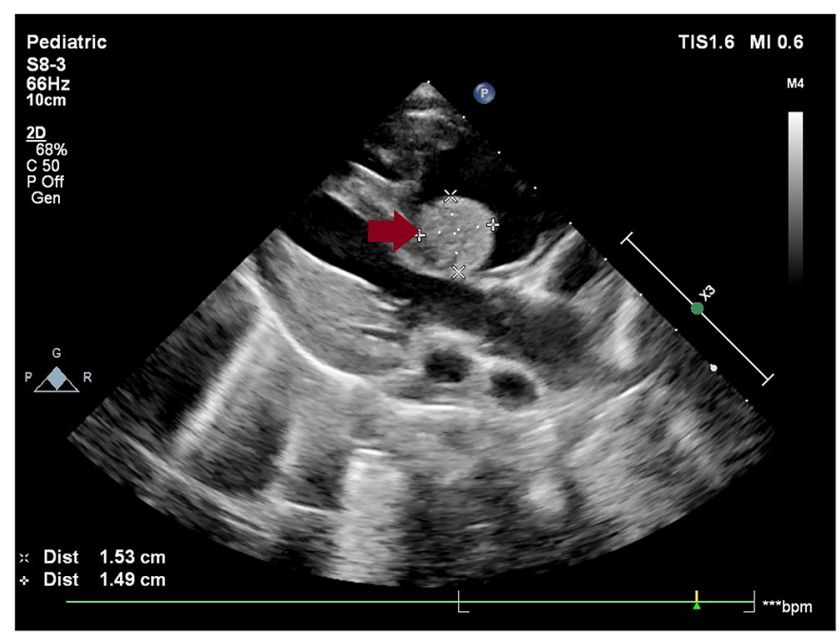

Fig. 1 Modified parasternal long-axis image of rhabdomyoma in TTE (red arrow)

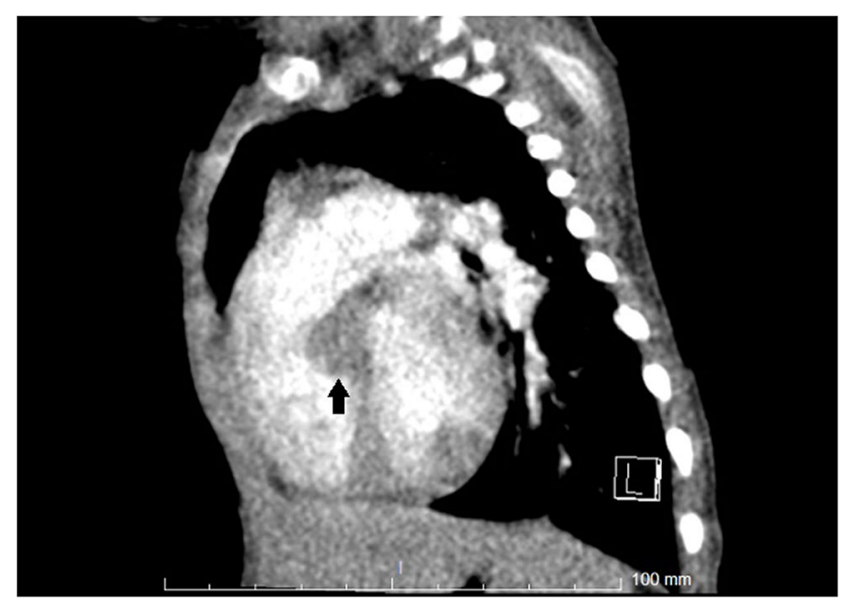

Fig. 2 Vertical long axis reformatted multidetector computed tomography image - rhabdomyoma is well defined (black arrow)

not excised because the primary lesion was rhabdomyoma and asymptomatic and did not cause functional and structural impairment. Additionally, in the case of resection, AV block risk was high. Subsequently, secundum ASD and right atriotomy were closed. Patient came off cardiopulmonary bypass (CPB) in sinus rhythm, albeit with dopamine supplementation at a dose of $5 \mathrm{mcg} / \mathrm{kg} / \mathrm{min}$.

Inotropic support was discontinued at the 4 th postoperative hour, and sedation support was discontinued at the 6th hour and child extubated at the 8th hour. The patient stayed in the intensive care unit for $22 \mathrm{~h}$ after extubation. The patient was followed up for 3 days in the ward and discharged on the 4th day uneventfully. Routine TTE performed before discharge showed an intact IVS with no residual defects. It was confirmed that the PDA was completely closed, and there was no regurgitation of $\mathrm{AV}$ valves.

After low-dose oral everolimus treatment $\left(0.4 \mathrm{mg} / \mathrm{m}^{2} /\right.$ day $)$, we observed that the tumor size decreased to $1.1 \times 0.8 \mathrm{~cm}$ and

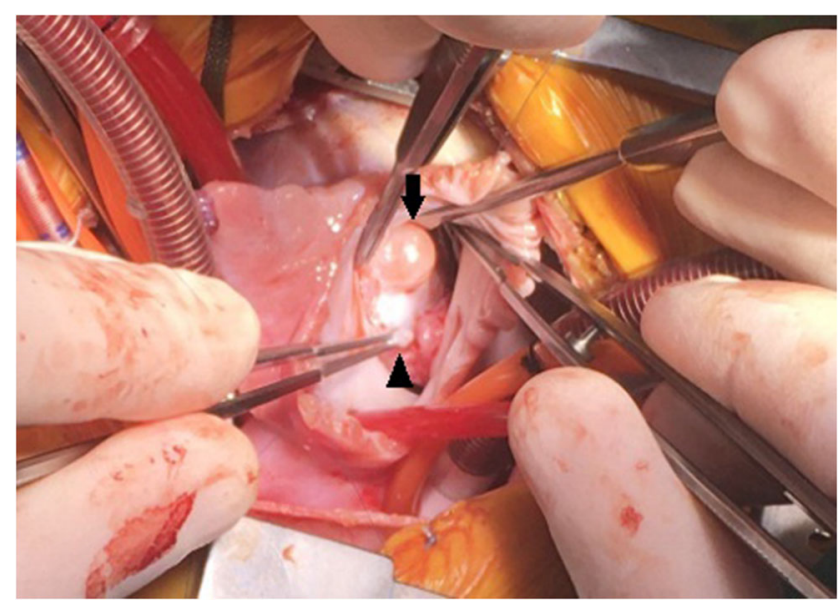

Fig. 3 The appearance of rhabdomyoma through the right atriotomy (black arrow), tricuspid valve anterior and septal leaflet (arrowhead) 
$0.9 \times 0.7 \mathrm{~cm}$ on TTE performed at the $3 \mathrm{rd}$ and 6 th months postoperatively, respectively.

\section{Discussion}

Primary cardiac tumors in childhood are extremely rare and are detected with a rate of $0.08 \%$ in autopsies and are mostly benign. The most common histologically seen infantile cardiac tumors are rhabdomyomas (60-85\%) and according to their incidence, teratoma, fibroma, hemangioma, and hamartomas, respectively [4-6]. In the last two decades, an increase in the incidence of pediatric cardiac tumors has been reported due to the increase in noninvasive imaging methods, the technological development of TTE devices, and the widespread use of them. Patients' admission complaints depend on the size and location of the mass, as they have an inlet or outlet obstruction and arrhythmogenic effect [7]. In general, the indications for resection are to eliminate hemodynamic and respiratory pathologies, if any, severe arrhythmia and a significant risk of embolization. These tumors are highly associated (60-80\%) with tuberous sclerosis complex (TSC). It originates from the free wall of the ventricle and ventricular septum. The diagnosis of rhabdomyoma is based on the characteristic TTE features of the tumor, in addition to the cutaneous, central nervous system, or renal manifestations of TS. In the TTE examination, they are seen as well-circumscribed, homogeneous, and echo bright masses [3]. Many studies have shown that rhabdomyomas that are medically followed or partially resected regress spontaneously within 1 to 3 years.

The patient we presented had AVSD and PDA accompanying rhabdomyoma. In the literature, the patient with congenital heart defect associated with TS and rhabdomyoma is very rare. In the study published by Jiang et al. [2], they suggested that the heart may disrupt the growth pattern in the region where the mass appeared during the embryological development phase. In our case, since the intrauterine cardiac examination was not performed, it cannot be clearly revealed in which trimester the rhabdomyoma occurred. However, the atrioventricular canal defect and the area where the rhabdomyoma is located are very close, suggesting that the tumor might cause the supposed development defect.

In the literature, it has been observed that the rhabdomyoma associated with the TSC is largely multiple. However, we observed a solitary rhabdomyoma in our case. In the study conducted by Bader et al. with 26 patients, the tumors in diagnosis were single in five patients (19\%), including the right or left ventricle and multiple in 21 patients (80.8\%) [8]. In a study conducted by Sciacca et al. with 33 patients, multiple rhabdomyomas were detected in all patients with TS [9]. AV channel defects often coexist with Down syndrome. However, in our case, the absence of Down syndrome is also interesting.
We did not excise rhabdomyoma in our patient since it was asymptomatic and was largely intramyocardial, and excision might have caused damage to the surrounding structures. Malignant arrhythmias and AV conduction blocks that may develop in the postoperative period after excision can be fatal. After birth, rhabdomyoma cells lose their ability to divide, and tumor regression in infancy is an expected result regardless of tumor size. In more than $80 \%$ of tumors, full regression may occur in early childhood. In addition, it has been revealed by many studies, everolimus, a mammalian target of rapamycin (mTOR) inhibitor, accelerates shrinkage in the size of rhabdomyomas by up to 12 times $[10,11]$. In our case, we observed a regression of the tumor with everolimus treatment in accordance with the literature.

\section{Conclusion}

In rare cases with congenital heart defects accompanying rhabdomyoma, we think that resection should not be preferred to preserve structural integrity, if the tumor is asymptomatic. We are of the opinion that excision of this cardiac tumor, which already lends to spontaneous regression, may negatively affect surgical success during the repair stages of other congenital defects. Everolimus treatment accelerates the regression process and makes it more beneficial to follow this tumor without excision.

Funding None

Data availability The data that support the findings of this study are available from the corresponding author (IM), upon reasonable request.

\section{Compliance with ethical standards}

Informed consent There is no patient identifiable data in the manuscript; hence, no informed consent is required.

Conflict of interest The authors declare that they have no conflicts of interest.

Ethical committee approval Not required.

Statement of Human and animal rights No animals involved.

Disclaimer The authors alone are responsible for the content and writing of the paper.

\section{References}

1. Watson GH. Cardiac rhabdomyomas in tuberous sclerosis. Ann N Y Acad Sci. 1991;615:50-7.

2. Jiang ZY, Pircova A, Sekarski N, et al. Transposition of the great arteries, pulmonary atresia, and multiple ventricular septal defects 
associated with multiple cardiac rhabdomyomas in a case of tuberous sclerosis. Pediatr Cardiol. 2000;21:165-9.

3. Hinton RB, Prakash A, Romp RL, Krueger DA, Knilans TK. Cardiovascular manifestations of tuberous sclerosis complex and summary of the revised diagnostic criteria and surveillance and management recommendations from the International Tuberous Sclerosis Consensus Group. J Am Heart Assoc. 2014;3:e001493.

4. Nadas AS, Ellison RC. Cardiac tumors in infancy. Am J Cardiol. 1968;21:363-6.

5. Tzani A, Doulamis IP, Mylonas KS, Avgerinos DV, Nasioudis D. Cardiac tumors in pediatric patients: a systematic review. World J Pediatr Congenit Heart Surg. 2017;8:624-32.

6. Allan LD, Hornberger LK, Sharland GK. Textbook of fetal cardiology. Greenwich Medical Media; 2000.

7. Yadava OP. Cardiac tumours in infancy. Indian Heart J. 2012;64: 492-6.
8. Bader RS, Chitayat D, Kelly E, et al. Fetal rhabdomyoma: prenatal diagnosis, clinical outcome, and incidence of associated tuberous sclerosis complex. J Pediatr. 2003;143:620-4.

9. Sciacca P, Giacchi V, Mattia C, et al. Rhabdomyomas and tuberous sclerosis complex: our experience in 33 cases. BMC Cardiovasc Disord. 2014;14:66.

10. Aw F, Goyer I, Raboisson M-J, Boutin C, Major P, Dahdah N. Accelerated cardiac rhabdomyoma regression with everolimus in infants with tuberous sclerosis complex. Pediatr Cardiol. 2017;38: 394-400.

11. Tiberio D, Franz DN, Phillips JR. Regression of a cardiac rhabdomyoma in a patient receiving everolimus. Pediatrics. 2011;127:e1335-e7.

Publisher's note Springer Nature remains neutral with regard to jurisdictional claims in published maps and institutional affiliations. 